\title{
Urolithins Attenuate Multiple Symptoms of Obesity in Rats Fed on a High-Fat Diet
}

This article was published in the following Dove Press journal:

Diabetes, Metabolic Syndrome and Obesity: Targets and Therapy

\author{
Abdulrasheed $\mathrm{O}$ \\ Abdulrahman (D) \\ Abudukadeer Kuerban $\mathbb{D}^{\prime}$ \\ Zuhair Ahmed Alshehri' \\ Wesam H Abdulaal' \\ Jalaluddin Awlia Khan' \\ Mohammad Imran Khan ${ }^{1,2}$ \\ 'Department of Biochemistry, Faculty of \\ Science, King Abdulaziz University, \\ Jeddah 21589, Saudi Arabia; ${ }^{2}$ Cancer \\ Metabolism and Epigenetic Unit, Faculty \\ of Science, King Abdulaziz University, \\ Jeddah 21589, Saudi Arabia
}

Background: Urolithins are gut microbiota-derived polyphenol metabolites, produced following the consumption of pomegranate, berries, and nuts. Recent studies have shown the potentials of these metabolites on reducing triglycerides accumulation in cultured hepatocytes and adipocytes. In this study, we investigated the ability of both urolithin A (Uro-A) and urolithin B (UroB) to attenuate obesity and associated symptoms in a high-fat diet-induced obesity model in rats. Methods: Twenty-four male Wistar rats were randomly assigned to four groups. Group 1 was fed on a normal diet while groups 2,3 , and 4 were fed on a high-fat diet for 10 weeks. After this, groups 3 and 4 were treated with $2.5 \mathrm{mg} / \mathrm{kg}$ body weight of Uro-A and Uro-B intraperitoneally, respectively. Body weight, serum lipid profile, hepatic antioxidant activity, hepatic lipid accumulation, fecal lipid content, and the expressions of genes involved in lipogenesis and hepatic ER stress were quantified.

Results: Indeed, a high-fat diet resulted in increased body weight, visceral adipose tissue mass, and oxidative stress in rats. However, treatment with both Uro-A and Uro-B decreased body weight and visceral adipose tissue mass. These metabolites restored hepatic antioxidant capacity and decreased lipid accumulation in addition to an increase in fecal fat excretion. Moreover, both Uro-A and Uro-B treatment downregulated the expression of LXR $\alpha$ and SREBP1c; involved in de novo lipogenesis while upregulating PPAR $\alpha$ expression for increased fatty acid oxidation. Furthermore, Uro-A and Uro-B decreased the expression of PERK and IRE1 $\alpha$; which are involved in hepatic ER stress. Taken together, our results showed the potentials of Uro-A and Uro-B in mitigating obesity symptoms and they could thus provide promising roles in the future as functional anti-obesity candidates.

Keywords: de novo lipogenesis, ER stress, high-fat diet, obesity, pomegranate, urolithins

\section{Introduction}

Obesity is a multifaceted disease that arises as a result of the interaction of dietary, environmental, lifestyle, and genetic factors, which tends toward a positive energy balance ie caloric intake exceeds energy expenditure. ${ }^{1}$ It is defined as a medical condition that is characterized by the abnormal accumulation of fat in the body and it is associated with hypertrophy (excessive growth) and hyperplasia (expansion) of adipose tissue as a result of the imbalance between energy intake and its expenditure. ${ }^{2}$ Increased oxidative stress caused by fat accumulation is a critical disease mechanism in obesity-associated metabolic syndrome. ${ }^{3}$ Obesity has been implicated as a contributing factor for a variety of health challenges such as cancer, type 2 diabetes, cardiovascular disease, and osteoarthritis. ${ }^{4}$

According to the World Health Organization, there are over 1.9 billion overweight people globally with obesity accounting for more than 650 million
Correspondence: Mohammad Imran Khan Department of Biochemistry, Faculty of Science, King Abdulaziz University, Jeddah 21589, Saudi Arabia

Tel +966-12-6952000

Email mikhan@kau.edu.sa
Diabetes, Metabolic Syndrome and Obesity: Targets and Therapy 2020:13 3337-3348

3337

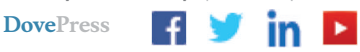

http://doiorg/10.2147/DMSO.s268146 
individuals. ${ }^{5}$ Given the prevailing health and economic burden of obesity, the discovery of economically viable means for obesity management becomes paramount. Current approaches include exercise, a decrease in body weight, and the use of pharmaceutics and other dietary intakes to target signaling pathways mediated by obesity. ${ }^{6}$ Moreover, some of the currently available anti-obesity pharmaceutical drugs are subject to being abuse, ${ }^{7}$ expensive with severe side effects, and not suitable for usage for a long period. ${ }^{8}$

New insight into the management of obesity involves the use of phenolic compounds. This is due to their ability to attenuate inflammation, drug resistance, and angiogenesis. They have also been reported to have anti-obesity property. ${ }^{9}$ Urolithins are dibenzopyran-6-one derived compounds having a different substitution of a hydroxyl group. ${ }^{10}$ They are naturally occurring secondary metabolites obtained through the gut microbial action on food that is abundant in ellagitannins and ellagic acids such as pomegranates, some berries, walnuts, and grapes. ${ }^{11}$ Urolithin A (Uro-A) and urolithin B (Uro-B), chemical name 3.8-dihydroxy-urolithin, and 3-hydroxy-urolithin respectively are two of the main urolithins found in circulation and tissues. ${ }^{12}$ Previous in vitro studies have reported the biological activities of urolithins such as antiinflammatory, antioxidant, antiglycative, anticancer, antimicrobial, and lipid-lowering property in addition to the improvement in mitochondrial and muscle function. ${ }^{13}$ These metabolites could serve to provide the evidence for the biological effects attributed to the intake of ellagitannin containing foods such as pomegranate. ${ }^{13}$

However, following the large intestinal absorption, urolithins are found in circulation as glucuronide, sulfate, and methylated metabolites between micromolar and nanomolar concentrations. ${ }^{14}$ In humans, different concentrations of glucuronides of Uro-A, Uro-B, and Uro-C were detected in plasma after the intake of ellagitannin rich food. These differences in concentration arise from the differential composition of the microbes that inhabit the gut. ${ }^{15}$ A previous study on Uro-A administration to rats showed its tissue distribution and that of its Phase II metabolites in organs such as the liver, colon, prostate, lung, intestine, and kidney. ${ }^{16}$ Uro-A and Uro-B glucuronides have also been detected in prostate biopsy. ${ }^{11}$

Several previous studies have reported the anti-obesity activity of pomegranate. These effects were seen in pomegranate juice, leaf extract, seed extract, and flower extract. ${ }^{17}$ Since pomegranate is a good source of ellagitannins, the biological activities of this fruit have been linked to urolithins. ${ }^{13}$ Besides, the urolithins have also been detected in the liver. ${ }^{16}$ Hence, we hypothesize that the reported lipid-lowering effect of urolithins points to a potential anti-obesity property and that the antiobesity effect reported for pomegranate might be mediated by urolithins.

In this study, we investigated the anti-obesity effects of urolithins (Uro-A and Uro-B) on high-fat diet-induced obesity model in rats and examined if these metabolites could reverse or reduce key metabolic changes in the liver arising from a high-fat diet (HFD).

\section{Materials and Methods \\ Diet}

Normal chow diet (ND) was purchased from Milling Company $2 \mathrm{MC} 2$, Riyadh, Saudi Arabia and contains $20 \%$ protein, $4 \%$ fat, $3.5 \%$ fiber, $6 \%$ ash, $64.4 \%$ carbohydrate, $1 \%$ calcium and $0.6 \%$ phosphorus.

The high-fat diet was prepared in the lab and composed of $40 \%$ normal diet, $40 \%$ cow lard, $10 \%$ sucrose, and $10 \%$ cholesterol.

\section{Animals}

Twenty-four male Wistar rats weighing $170 \pm 3.0 \mathrm{~g}$ were purchased from the animal house of King Fahd Medical and Research Centre (KFMRC), King Abdulaziz University, Jeddah, Saudi Arabia. Animals were acclimatized on a normal diet for 1 week at a temperature of $22-24{ }^{\circ} \mathrm{C}$ and on a 12-hour light and dark cycle. Following acclimatization, animals were randomly divided into 4 groups ( $n=6$ per group).

Group 1 (ND), control group, were fed on a normal diet, and Group 2, 3, and 4 were fed on HFD for 10 weeks with weekly body weight measurement. The animals in the high-fat diet group were considered obese when their body weight was $10 \%$ higher than the weight of animals in the control group. $^{18,19}$

\section{Treatment}

Urolithin A and B (97\% purity) were purchased from BLD Pharma, China. After the 10 weeks of feeding, animals in group 3 HFDA (HFD + Uro-A) and group 4 HFDB (HFD + Uro-B) were given an intraperitoneal (I.P.) injection of Uro-A and Uro-B at a dose of $2.5 \mathrm{mg} / \mathrm{kg}^{12}$ four times a week for 4 weeks. 
The urolithin stock solution was prepared by dissolving the compounds in dimethyl sulfoxide (DMSO) at a concentration of $20 \mathrm{mg} / \mathrm{mL}$ and stored at $4{ }^{\circ} \mathrm{C}$ in the dark. Before I. P. injection, an aliquot of the stock solution was diluted in phosphate-buffered saline (PBS) and the required concentration of urolithin was injected into the animals with a final DMSO blood concentration less than $0.5 \%$. Animals in the ND group, as well as the HFD group, received the same volume of DMSO. Since urolithins are derived from the gut microbiota transformation of ellagitannins, in this study, we have used the synthetic forms of the compounds and hence, the microbiota transformation step for urolithin formation is not required. In doing so, we believe that; first, the I. $P$. injection ensures that the gastrointestinal tract is bypassed thereby ensuring that the redundant catabolism of Uro-A and Uro-B is avoided. Second, I.P. injection ensures that Uro-A and Uro-B enter directly through the portal vein followed by a hepatic first-pass effect before reaching systemic circulation. ${ }^{20}$ And last, DMSO could irritate the animals as a vehicle for urolithins when administered orally, administration of these compounds through intraperitoneal injection will be less irritating to the animals.

In the end, animals were fasted overnight, euthanized under diethyl ether anesthesia, and blood was taken from the abdominal aorta. Tissues were excised, rinsed in PBS, and weighed. Tissues were frozen at $-80{ }^{\circ} \mathrm{C}$ or stored in $10 \%$ buffered formalin for histological analysis. All experiments involving animals were done according to the United States National Institute of Health's revised Guide for the Care and Use of Laboratory Animals and approved by the Bioethics Committee of the Department of Biochemistry, Faculty of Science, King Abdulaziz University (No: 1013096).

\section{Determination of Biochemical}

\section{Parameters}

Serum samples were obtained from whole blood after centrifugation at $3000 \mathrm{rpm}$ for $10 \mathrm{~min}$. Samples were stored at $-80{ }^{\circ} \mathrm{C}$ until use. Serum levels of aspartate aminotransferase (AST), alkaline phosphatase (ALP), total cholesterol, triglyceride (TG), high-density lipoprotein cholesterol (HDL-C) were quantified with a commercial kit (Crescent diagnostics, Jeddah, Saudi Arabia).

\section{Preparation of Liver Homogenate}

Ten percent (10\%) liver homogenate was prepared in 100 $\mathrm{mM}$ phosphate buffer ( $\mathrm{pH}$ 7.4) containing $1 \mathrm{mM}$ EDTA and centrifuged at $10,000 \mathrm{rpm}$ for $15 \mathrm{~min}$ at $4{ }^{\circ} \mathrm{C}$. Then, the supernatant was taken and stored at $-80{ }^{\circ} \mathrm{C}$ until analysis. Protein content in the supernatant was accessed by the method of Bradford. ${ }^{21}$

\section{Determination of Liver Antioxidant Enzymes Activities}

Hepatic catalase activity was determined by monitoring the decomposition of $\mathrm{H}_{2} \mathrm{O}_{2}$ according to the method of Aebi. ${ }^{22}$ Briefly, $50 \mu \mathrm{L}$ of supernatant, $500 \mu \mathrm{L}$ of $\mathrm{H}_{2} \mathrm{O}_{2}(30$ $\mathrm{mM}$; pH 7.0), $1950 \mu \mathrm{L}$ phosphate buffer (100 mM; pH 7.0) and $500 \mu \mathrm{L}$ of distilled water in a total reaction volume of $3 \mathrm{~mL}$. A change in absorbance was recorded for $1 \mathrm{~min}$ at $240 \mathrm{~nm}$ using a quartz cuvette. The molar extinction coefficient of $\mathrm{H}_{2} \mathrm{O}_{2}$ was taken as $39.4 \mathrm{M}^{-1} \mathrm{~cm}^{-1}$ and activity expressed as $\mu \mathrm{mol}$ of $\mathrm{H}_{2} \mathrm{O}_{2}$ decomposed per min per mg protein.

Hepatic superoxide dismutase (SOD) activity was assayed by assessing its superoxide ion scavenging activity produced from the auto-oxidation of pyrogallol as described previously. ${ }^{23}$ Briefly, $50 \mu \mathrm{L}$ of supernatant was added to $2900 \mu \mathrm{L}$ Tris- $\mathrm{HCl}(50 \mathrm{mM}$; $\mathrm{pH} 7.4$ containing 1 mM EDTA) in a quartz cuvette. The reaction was initiated against a Tris- $\mathrm{HCl}$ blank by the addition of $50 \mu \mathrm{L}$ pyrogallol solution $(60 \mathrm{mM}$ in $1 \mathrm{M} \mathrm{HCl})$ and absorbance recorded at $325 \mathrm{~nm}$ for every $30 \mathrm{sec}$ for $5 \mathrm{~min}$.

$$
\text { \%inhibition }=\frac{\left(\frac{\Delta A_{\text {control }}}{T}\right)-\left(\frac{\Delta A_{\text {sample }}}{T}\right)}{\left(\frac{\Delta A_{\text {control }}}{T}\right)} \times 100 \%
$$

where $\Delta \mathrm{A}_{\text {control }}$ is the change in absorbance at $325 \mathrm{~nm}$ of the mixture without the sample (Tris- $\mathrm{HCl}+$ pyrogallol) and $\Delta \mathrm{A}_{\text {sample }}$ is a change in absorbance at $325 \mathrm{~nm}$ of the mixture containing the sample; $\mathrm{T}=5 \mathrm{~min}$.

\section{Determination of Liver Levels of Glutathione (GSH) and Lipid Peroxidation} The liver level of GSH was determined using a commercial kit (Biodiagnostic, Egypt), according to the manufacturer's instruction.

The effect of HFD on lipid peroxidation in liver homogenates was quantified by measuring the malondialdehyde (MDA) formed using the thiobarbituric acid-reactive substances (TBARS) assay according to the method of Ohkawa et al. ${ }^{24}$ Briefly, $500 \mu \mathrm{L}$ of $10 \% \mathrm{w} / \mathrm{v}$ liver homogenate was prepared in $100 \mathrm{mM}$ phosphate buffer containing $1 \mathrm{mM}$ EDTA. $1 \mathrm{~mL}$ of TBA:TCA: $\mathrm{HCl}$ reagent $(0.38 \%$ thiobarbituric acid prepared in distilled water and warmed 
in a water bath, $15 \%$ trichloroacetic acid dissolved in distilled water, and $0.25 \mathrm{~N}$ hydrochloric acid, ratio $1: 1: 1)$ was then added to the homogenate, boiled at $90{ }^{\circ} \mathrm{C}$ for 20 min, and cooled on ice. Following centrifugation at $1000 \mathrm{rpm}$ for $10 \mathrm{~min}$, the absorbance of TBARS was measured at $532 \mathrm{~nm}$ against a reagent blank. The MDA content in liver homogenate was thus calculated as TBARS and expressed as nmol/g tissue using its molar extinction coefficient of $1.56 \times 10^{5} \mathrm{M}^{-1} \mathrm{~cm}^{-1}$.

\section{Liver and Fecal Lipids Content}

Liver and fecal lipid contents were extracted according to the Folch method. ${ }^{25}$ Briefly, frozen liver and fecal samples (100 mg) were homogenized completely and separately in $1 \mathrm{~mL}$ of chloroform: methanol (2:1) respectfully followed by the addition of $200 \mu \mathrm{L}$ of deionized water. Each mixture was vortexed vigorously followed by centrifugation at $14,000 \mathrm{~g}$ for $10 \mathrm{~min}$ to generate a lower organic and upper aqueous phase. From the lower phase, $200 \mu \mathrm{L}$ was taken into an already weighed $1.5 \mathrm{~mL}$ centrifuged tube, then dried using a vacuum centrifuge at $1300 \mathrm{rpm}$ for $2 \mathrm{hrs}$ at $37^{\circ} \mathrm{C}$. The leftover lipid was then weighed. From the hepatic lipid, the dried lipids were subsequently dissolved in $50 \mu \mathrm{L}$ of $100 \%$ isopropanol. The hepatic TG and cholesterol contents were quantified using a commercial assay kit (Crescent diagnostics, Jeddah, Saudi Arabia) with values normalized to tissue weight in gram.

\section{RNA Extraction and RT-qPCR}

Total RNA from the liver tissue was extracted using PureLink RNA Mini Kit (Invitrogen) according to the manufacturer's instructions and $2 \mu \mathrm{g}$ total RNA was reversed transcribed into cDNA with High Capacity cDNA Reverse Transcription kit (Applied Biosystem) using MultiGene Labnet thermal cycler (Labnet International inc). For primer design, coding sequence was obtained from the Ensembl genome browser and primers were picked using Primer3 software. To confirm the specific binding of the primers, In-silico PCR was done using the UCSC genome browser.

Real-time quantitative PCR was carried out using Power up SYBR Green Master Mix (Applied Biosystem) in a 7300 Real-Time PCR system. The total reaction mixture $(10 \mu \mathrm{L})$ contains $5 \mu \mathrm{L} 2 \mathrm{X}$ SYBR Green master mix, $1 \mu \mathrm{L}$ forward primer, $1 \mu \mathrm{L}$ reverse primer, $2 \mu \mathrm{L}$ DEPC- $\mathrm{H}_{2} \mathrm{O}$, and $1 \mu \mathrm{L}$ cDNA. PCR reaction was done in a cycling protocol of $95^{\circ} \mathrm{C}$ for $2 \mathrm{~min}$, followed by 40 PCR cycles of denaturation at 95 ${ }^{\circ} \mathrm{C}$ for $15 \mathrm{~s}$ and $60^{\circ} \mathrm{C}$ for $1 \mathrm{~min}$ for annealing and elongation.
The relative mRNA expression levels were analyzed according to the $2^{-\Delta \Delta \mathrm{CT}} \operatorname{method}^{26}$ and normalized to the expression of the ( $\beta$-actin). The primer sequences are given in supplementary Table 1.

\section{Histopathological Examination}

Liver tissues were fixed in 10\% neutral-buffered formalin solution, followed by dehydration in graded alcohol, clearance in xylene, and finally embedded in paraffin for 12 hours. Paraffin blocks were sectioned at $5-\mu \mathrm{m}$ thickness using a microtome. Slides were stained with hematoxylin and eosin (H\&E) solution. Images were taken using an Olympus BX 51 light microscope.

\section{Statistical Analysis}

Data are presented as mean $\pm \mathrm{SE}$. Plot fitting and statistical analysis with one-way ANOVA followed by Dunnett's multiple comparisons test were performed using GraphPad Prism V6.0 software (GraphPad Software, San Diego, CA). Kolmogorov-Smirnov D test was used to check for normality. Different levels of significance are denoted as follows: ${ }^{*} \mathrm{p}<0.05 ; * * \mathrm{p}<0.01 ; * * * \mathrm{p}<0.001$; $* * * * \mathrm{p}<0.0001$

\section{Results}

\section{Urolithins Decreased Final Body Weight of Rats Fed on a High-Fat Diet}

In this study, we have used I.P. injection of Uro-A and Uro-B at a dose of $2.5 \mathrm{mg} / \mathrm{kg}$ body weight. Using the conversion factor of $6.17^{27}$ this is equivalent to a human dose of $0.45 \mathrm{mg} / \mathrm{kg}$ body weight. For a $60 \mathrm{~kg}$ human, it is $24.3 \mathrm{mg}$ of free Uro-A/Uro-B or about $44 \mathrm{mg}$ of Uro-A glucuronide/Uro-B glucuronide which should exist in circulation to predict similar biological effects found in the high-fat diet rats. A $60 \mathrm{~kg}$ adult has about $5 \mathrm{~L}$ of blood ${ }^{28}$ and assuming that the glucuronide conjugates gained full access to the internal volume of the human blood cells, $44 \mathrm{mg}$ of these conjugates will be equal to a plasma concentration of $22 \mu \mathrm{M}$ of Uro-A glucuronide or $23 \mu \mathrm{M}$ of Uro-B glucuronide respectively. These concentrations are within previously found range of $0.024 \mu \mathrm{M}$ to $35 \mu \mathrm{M}$ in human plasma especially for Uro-A glucuronide. ${ }^{29}$

The final body weight $(\mathrm{p}<0.05)$, the liver $(\mathrm{p}<0.05)$ and visceral adipose tissue mass (epididymal + perirenal fats) ( $p<0.0001$ ) of animals fed on an HFD were significantly higher when compared to animals fed on a normal diet (Table 1 and Figure 1). However, the 
Table I Final Body Weight and Biochemical Parameters of Experimental Animals

\begin{tabular}{|l|l|l|l|l|}
\hline Parameter/Group & ND & HFD & HFDA & HFDB \\
\hline Final body weight (g) & $335.0 \pm 13.8^{\mathrm{b}}$ & $430.0 \pm 18.6$ & $350.0 \pm 26.0^{\mathrm{a}}$ & $340.0 \pm 24.5^{\mathrm{a}}$ \\
AST (U/L) & $161.5 \pm 2.2$ & $153.3 \pm 7.4$ & $104.3 \pm 2.9^{\mathrm{a}}$ & $97.9 \pm 9.7^{\mathrm{a}}$ \\
ALP (U/L) & $103.7 \pm 3.6$ & $120.2 \pm 7.2$ & $100.7 \pm 10.4$ & $104.0 \pm 11.0$ \\
Triglycerides (mg/dL) & $62.1 \pm 5.6^{\mathrm{d}}$ & $138.9 \pm 6.5$ & $71.2 \pm 10.3^{\mathrm{c}}$ & $65.6 \pm 10.1^{\mathrm{c}}$ \\
Cholesterol (mg/dL) & $54.6 \pm 4.2^{\mathrm{g}}$ & $71.9 \pm 3.3$ & $55.22 \pm 2.0^{\mathrm{e}}$ & $49.7 \pm 3.2^{\mathrm{f}}$ \\
HDL-cholesterol (mg/dL) & $37.8 \pm 2.2^{\mathrm{b}}$ & $25.9 \pm 1.8$ & $37.6 \pm 2.8^{\mathrm{a}}$ & $38.3 \pm 5.0^{\mathrm{a}}$ \\
\hline
\end{tabular}

Notes: Data presented as mean \pm SE $(n=6)$. Letters bearing the same and different superscripts within a row are significantly different compared to HFD according to oneway ANOVA followed by Dunnett's multiple comparisons test. ${ }^{*} \mathrm{p}<0.05$.

treatment of animals with both urolithin A $(\mathrm{p}<0.05)$ and $\mathrm{B}(\mathrm{p}<0.05)$ significantly decreased the final body weight respectively when compared with untreated animals fed on an HFD. Similarly, Uro-A $(\mathrm{p}<0.01)$ and Uro-B $(\mathrm{p}<0.01)$ significantly decreased the visceral adipose tissue mass when compared with untreated animals fed on an HFD. While Uro-A effect on liver weight was not significant, Uro-B treatment on the other hand, significantly $(\mathrm{p}<0.05)$ decreased the liver weight when compared to untreated animals fed on a high-fat diet (Figure 1).

\section{Urolithins Mediate Positive Effects on Serum Biochemical Parameters}

The serum cholesterol $(\mathrm{p}<0.01)$ and TG $(\mathrm{p}<0.0001)$ levels were significantly elevated in animals fed on an HFD as compared to animals fed on a normal diet. Treatment with both Uro-A and B both decreased serum cholesterol $(p<0.01)$ and TG $(p<0.0001)$ levels respectively when compared to untreated animals fed on an HFD (Table 1). HFD results in a significant decrease $(\mathrm{p}<0.05)$ in the HDL level when compared to animals on a normal diet. However, Uro-A and Uro-B treatment both significantly elevated serum HDL level $(p<0.05)$ when compared to untreated animals fed on an HFD. Furthermore, there was no significant difference in the levels of serum ALP amongst the group. However, Uro$A$ and Uro-B treatment significantly decreased serum AST level $(\mathrm{p}<0.05)$ when compared with untreated animals fed on an HFD (Table 1).

\section{Urolithins Revealed Antioxidant Activity in vivo}

In previous reports, the consumption of HFD was well associated with elements of oxidative stress in various tissues including the liver. ${ }^{30}$ In agreement with previous studies, we observed that HFD resulted in an elevated hepatic level of TBARS $(p<0.05)$ when compared to animals fed with a normal diet (Figure 2). Both Uro-A $(\mathrm{p}<0.01))$ and Uro-B $(\mathrm{p}<0.05)$ treatment decreased these values when compared to untreated animals fed on an HFD. High-fat diet feeding resulted in a decrease in the GSH content when compared to animals fed on a normal diet but ( $p>0.05$ ). Both Uro-A and Uro-B elevated the GSH content when compared to untreated animals fed on an HFD but $(\mathrm{p}>$ $0.05)$. Similarly, HFD led to a significant decrease $(\mathrm{p}<$ 0.05 ) in the activities of catalase and SOD respectively when compared to animals fed on a normal diet (Figure 2). Although Uro-B treatment increased the activities of both catalase and SOD when compared
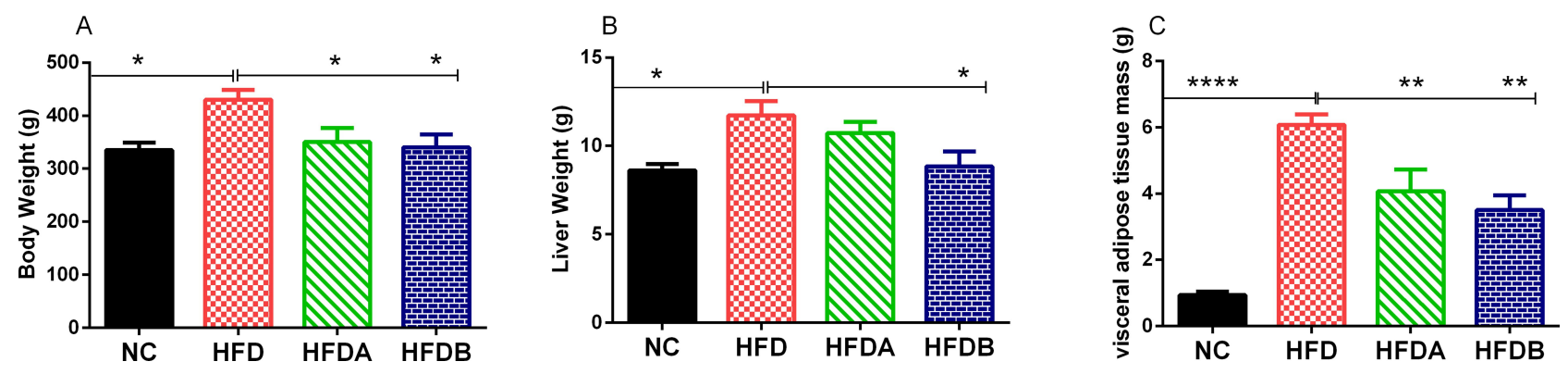

Figure I Effects of Uro-A and Uro-B on body weight and weight of organs. Final body weight $(\mathbf{A})$, liver weight (B), adipose tissue mass $(\mathbf{C})$. Data presented as mean \pm SE $(n=6) . * p<0.05 ; * * p<0.01 ; * * * *<0.0001$ against HFD. 

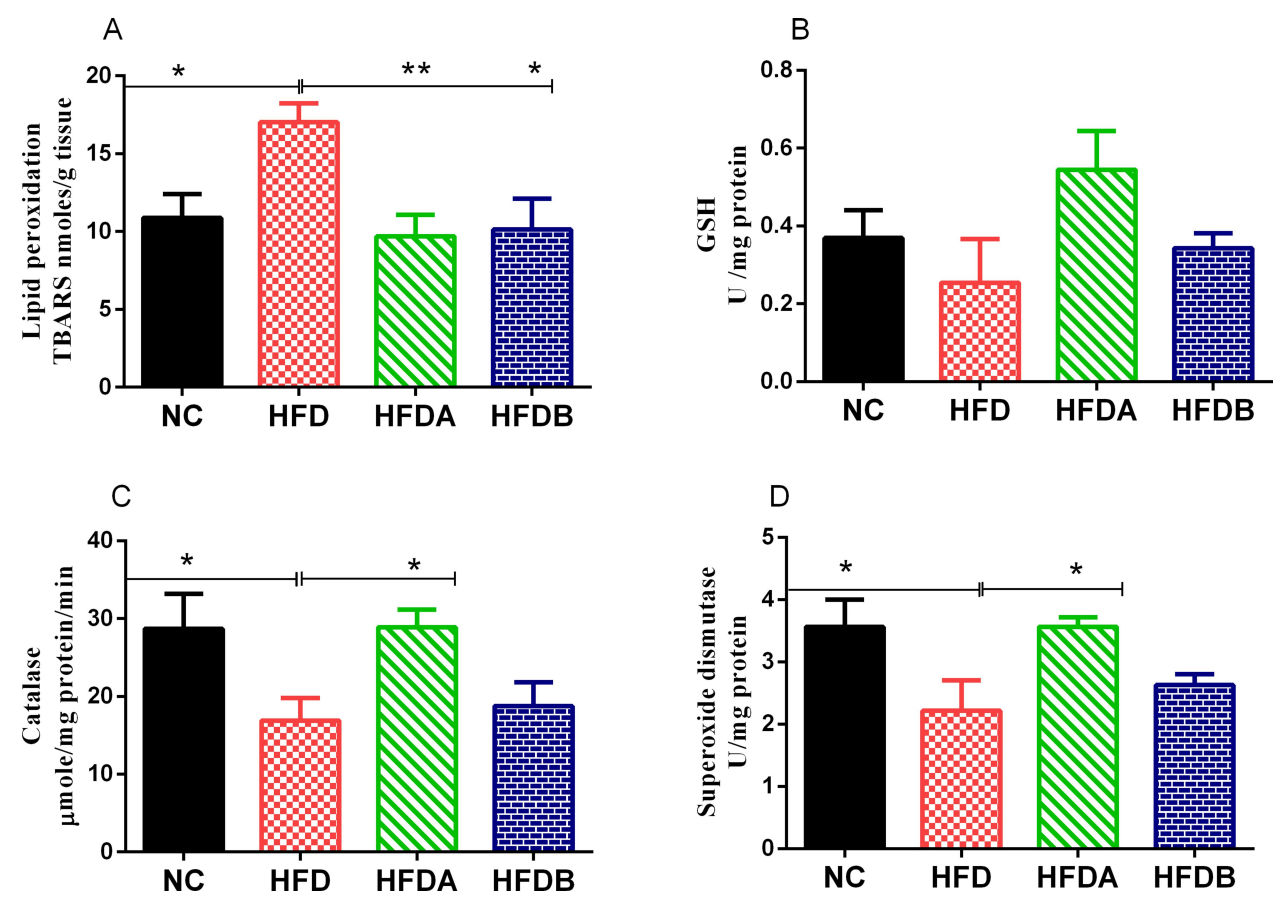

Figure 2 Effects of Uro-A and Uro-B on hepatic antioxidant status and activity. Lipid peroxidation (A), glutathione content (B), catalase activity (C), superoxide dismutase activity (D). Data presented as mean $\pm \mathrm{SE}(\mathrm{n}=6) .{ }^{*} \mathrm{p}<0.05 ;{ }^{* *} \mathrm{p}<0.01$ against HFD.

to untreated animals fed on HFD but ( $\mathrm{p}>0.05)$, Uro-A on the other hand, significantly increased the activities of these enzymes $(\mathrm{p}<0.05)$ when compared to untreated animals fed on HFD.

\section{Urolithins Attenuated Hepatic Lipid Levels, Increased Fecal Fat Excretion and Restored Liver Histology}

The total hepatic lipids $(\mathrm{p}<0.0001)$, triglyceride $(\mathrm{p}<$ $0.0001)$, and cholesterol $(\mathrm{p}<0.01)$ levels were significantly higher in animals fed an HFD as compared to the normal diet-fed animals (Figure 3). Treatment with both Uro-A $(\mathrm{p}<0.0001))$ and Uro-B $(\mathrm{p}<0.001)$ resulted in a decrease in total hepatic lipids (Figure 3A). Similarly, we observed a decline in the hepatic TG levels in rats treated with both Uro-A $(p<0.0001)$ and Uro $B(p<0.001)$ when compared to untreated HFD rats (Figure 3B). Furthermore, both Uro-A ( $<<$ $0.05)$ and Uro-B $(p<0.05)$ resulted in a significant decrease in hepatic cholesterol levels (Figure 3C). Furthermore, Uro-A $(\mathrm{p}<0.01)$ and Uro-B $(\mathrm{p}<0.05)$ treatment significantly increase fecal lipid excretion as compared to untreated animals fed on an HFD (Figure 3D). Histologically, HFD results in a visible and aggressive hepatic lipid accumulation (Figure 4) which was not observed in the normal diet-fed animals. This hepatic lipid accumulation was attenuated by treatment with Uro-A and Uro-B.

\section{Urolithins Modulate the Expression of Genes Involved in De Novo Lipogenesis and Hepatic ER Stress}

High-fat diet feeding resulted in a 1.2 fold increase in the expression levels of LXR $\alpha$ when compared to control animals fed on a normal diet and treatment with both Uro-A $(\mathrm{p}<0.05)$ and Uro-B $(\mathrm{p}<0.05)$ downregulated LXR $\alpha$ mRNA expressions respectively when compared to untreated animals fed on HFD (Figure 5). Similarly, an HFD significantly upregulated the mRNA expression of SREBP1c when compared to the normal diet-fed animals. Both Uro-A $(p<0.01)$ and Uro-B ( $p$ $<0.001)$ treatment significantly downregulated the expression of SREBP1c respectively when compared to untreated animals fed on HFD. In addition to this, both Uro-A $(p<0.05)$ and $B(p<0.05)$ upregulated the expression of PPAR $\alpha$ when compared to untreated animals fed on an HFD. Furthermore, compared with untreated HFD rats, Uro-A $(p<0.05)$ and Uro$B(p<0.05)$ treated rats have downregulated expression levels of PERK and IRE1 $\alpha$ respectively (Figure 5). These results showed that urolithins repressed the de-novo lipogenesis in the liver of HFD fed rats. 
A

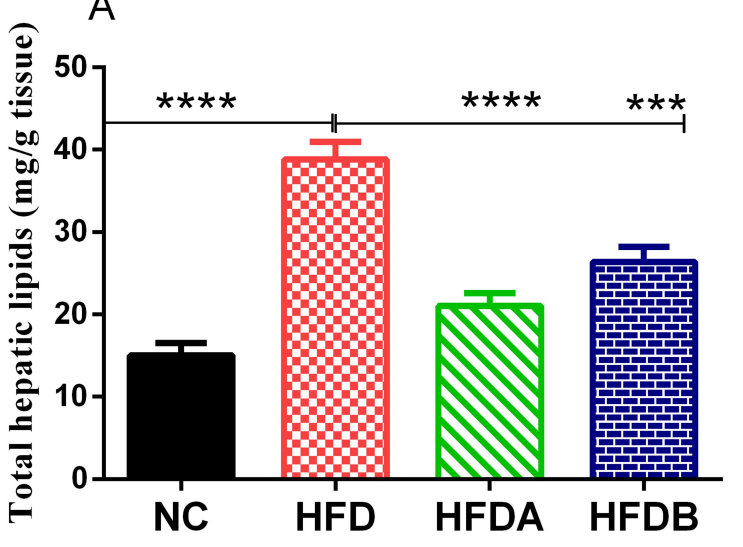

C

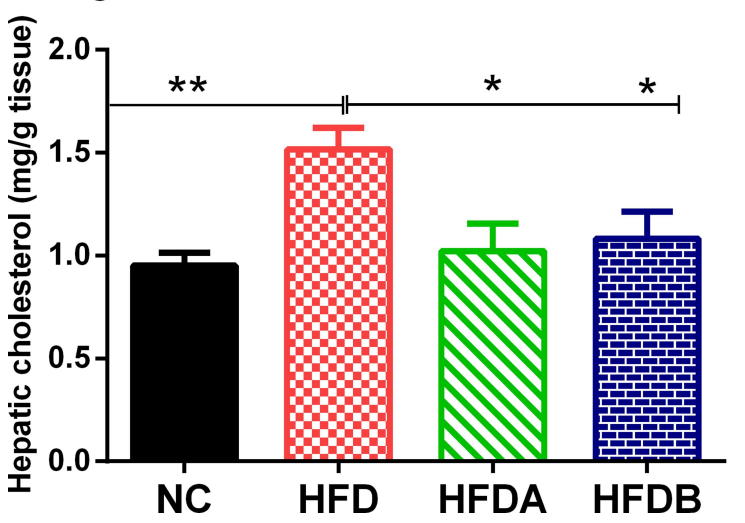

B

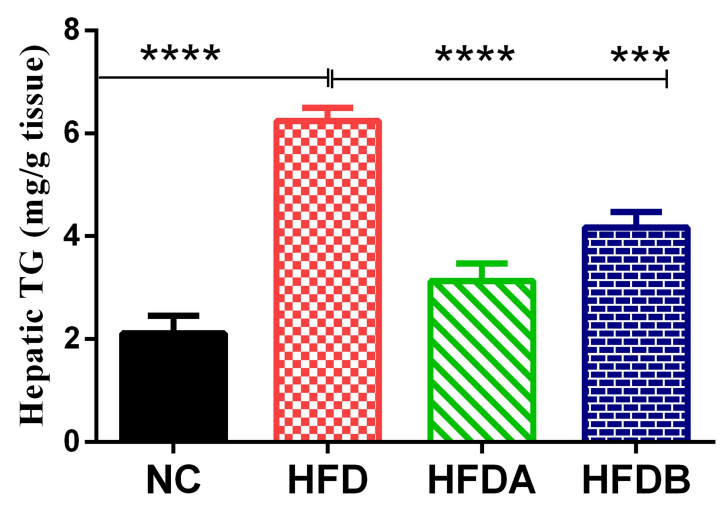

D

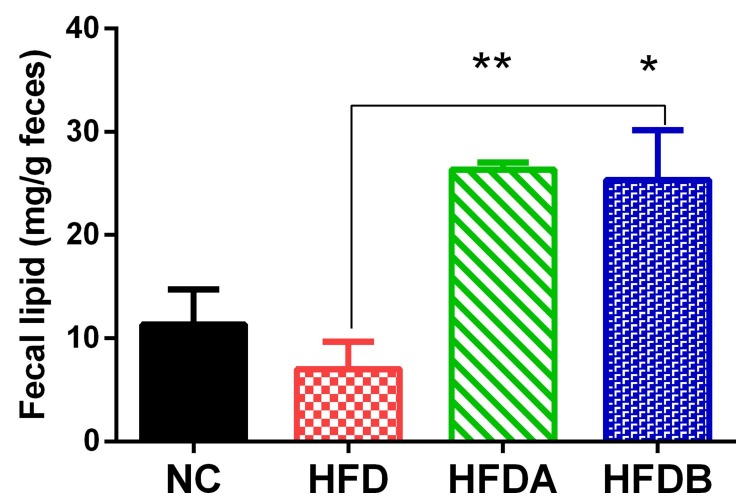

Figure 3 Effects of Uro-A and Uro-B on hepatic lipids accumulation and fecal lipids excretion. Total hepatic lipids (A), hepatic TG content (B), hepatic cholesterol (C), fecal lipids (D). Data presented as mean \pm SE $(n=6) .{ }^{*} p<0.05$; ${ }^{* *} p<0.01$; ${ }^{* * *} p<0.00$ I; ${ }^{* * * *} p<0.000$ I against HFD.

\section{Discussion}

Obesity is a metabolic disease associated with various health challenges. ${ }^{31}$ Several previous studies suggest that the consumption of food sources rich in polyphenols for a long duration could be protective against obesity and associated metabolic complications such as cardiovascular disease, type 2 diabetes and non-alcoholic fatty liver disease. $^{32,33}$

The anti-obesity effect of a compound or a drug candidate may act through either the stimulation of lipolysis, inhibition of lipogenesis, suppression of appetite, reduction in lipid absorption in the blood, or induction of thermogenesis. ${ }^{34}$ In this study, we investigated the antiobesity effect of Uro-A and B and their ability to modulate the symptoms associated with obesity in high-fat dietinduced obese rats. Our results suggest that not only do these gut-derived metabolites of ellagitannins decreased the final body weights of animals fed with HFD; they also decreased the visceral adipose tissue mass. These metabolites likely mediate this increase in weight loss as a result of an increase in the rate of fatty acid oxidation, decreased lipogenesis, and reduction in lipid accumulation and absorption.

Undoubtedly, the liver is an important organ for the metabolism of lipids. The build of unwanted fat in this organ serves as a predisposing factor for the development of hepatic disease. ${ }^{35}$ Thus, oxidative damage to the liver due to insults from reactive oxygen species (ROS) generated from a high-fat diet becomes increasingly elevated when there is a decline in the activities of antioxidant enzymes such as superoxide dismutase and catalase. ${ }^{36}$ Our results showed that Uro-A and Uro-B differentially impacted on the antioxidant status of animals fed with an HFD. Indeed, Uro-A unlike Uro-B increased the catalase and superoxide anion scavenging activities (Figure 2). However, they both significantly reduced lipid peroxidation. These effects confirmed an earlier report on the antioxidant activities of urolithins. ${ }^{15}$ Interestingly, our results 


\section{ND}

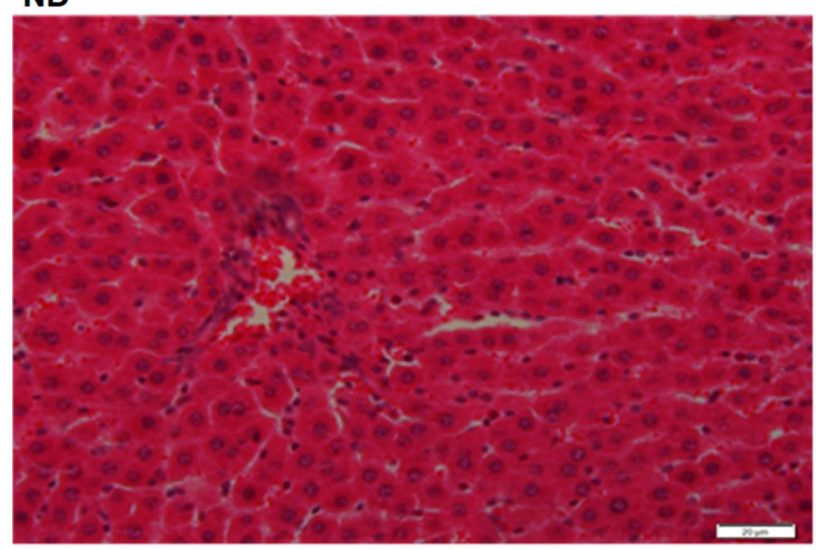

HFD

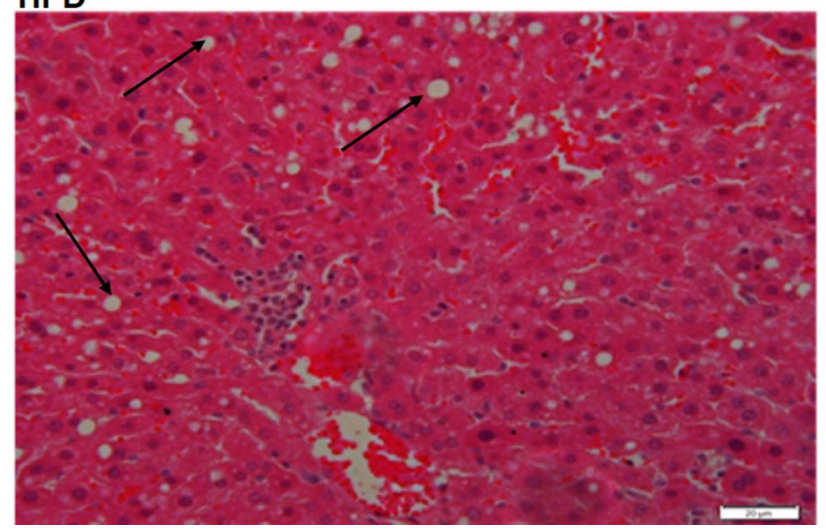

\section{HFDA}

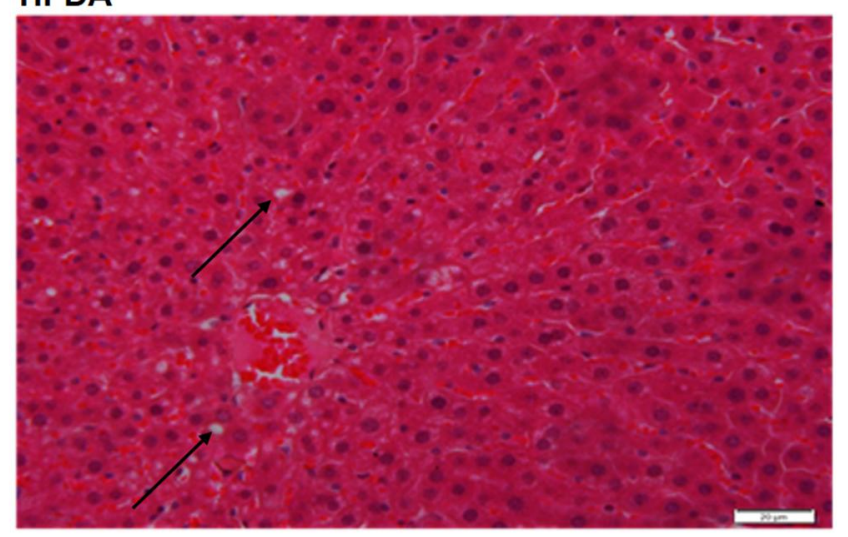

HFDB

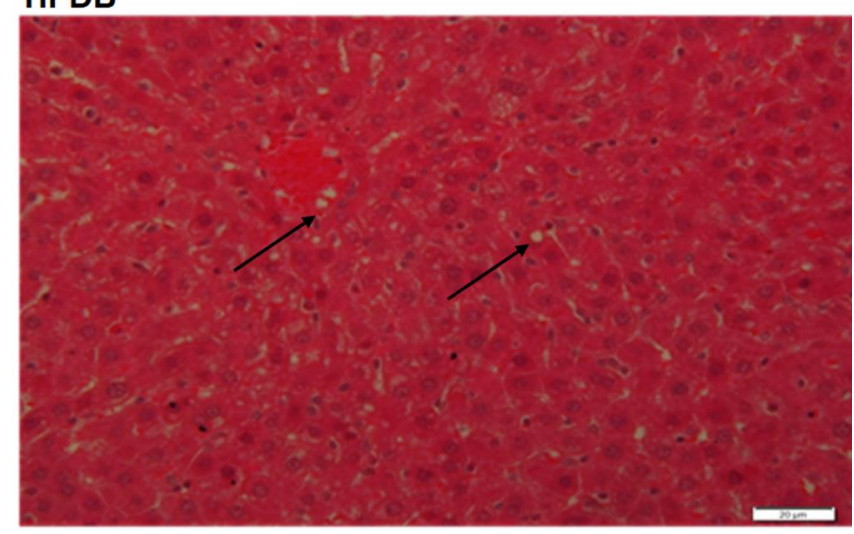

Figure 4 Effects of Uro-A and Uro-B on hepatic histology on lipids accumulation. Representative histological sections of the liver following H\& E staining (400x), scale bar, $20 \mu \mathrm{m}$. Arrow showing fat droplet. ND (normal diet-fed animals), HFD (untreated high-fat diet-fed animals), HFDA (Uro-A treated animals fed on a high-fat diet, HFDB (Uro$B$ treated animals fed on a high-fat diet).

showed that Uro-A has a superior antioxidant activity as compared to Uro B (Figure 2). This is likely due to the presence of two hydroxyl functional groups on the Uro-A ring (Figure 6).

A high-fat diet is responsible for high hepatic cholesterol and TG levels in the animal model. ${ }^{37}$ Previous studies have shown that urolithin A compared to Uro-B, significantly decreased triglycerides accumulation in cultured hepatocytes and adipocytes. ${ }^{38}$ Intriguingly, another study showed that Uro-B decreased lipid plaques deposition in double knockout apoE mice. ${ }^{39}$ In our study, both urolithins significantly decreased serum cholesterol and TG levels (Table 1). They also significantly decreased both hepatic cholesterol and TG levels (Figure 3), signifying the hypolipemic effect of Uro-A and Uro-B in vivo. Furthermore, both Uro-A and Uro-B significantly increased fecal lipid excretion (Figure 3). We speculate that the total lipid-lowering effects of these metabolites might involve either the increase clearance of both hepatic and serum cholesterol into secretion in bile or through the increased hepatic lipid excretion in the feces. Thus, the urolithin inhibitory effect on TG absorptions could serve as a potential strategy for weight loss since an excess accumulation of dietary TG is linked to obesity. ${ }^{40}$

Orlistat, a pharmacologically available drug used in the management of obesity functions primarily by reducing dietary fat absorption in the blood and increasing fecal fat excretion. ${ }^{41}$ Interestingly, our results on the effect of urolithin on serum lipid profile and fecal fat showed a similar trend. It is possible that the Uro-A and Uro-B mechanism of action in reducing body weight gain partly involve the increase in fecal fat excretion and reduction in dietary fat absorption.

In addition to weight reduction, another strategy for the control of accumulation of hepatic lipids and hence obesity is the inhibition of fat synthesis through lipogenesis and an increase in the breakdown of fat through beta-oxidation. We sought to understand the effect of urolithins on the expression level of genes related to hepatic lipogeneses and lipolysis. Liver $\mathrm{X}$ receptors $(\mathrm{LXR} \alpha$ and $\mathrm{LXR} \beta)$ are 

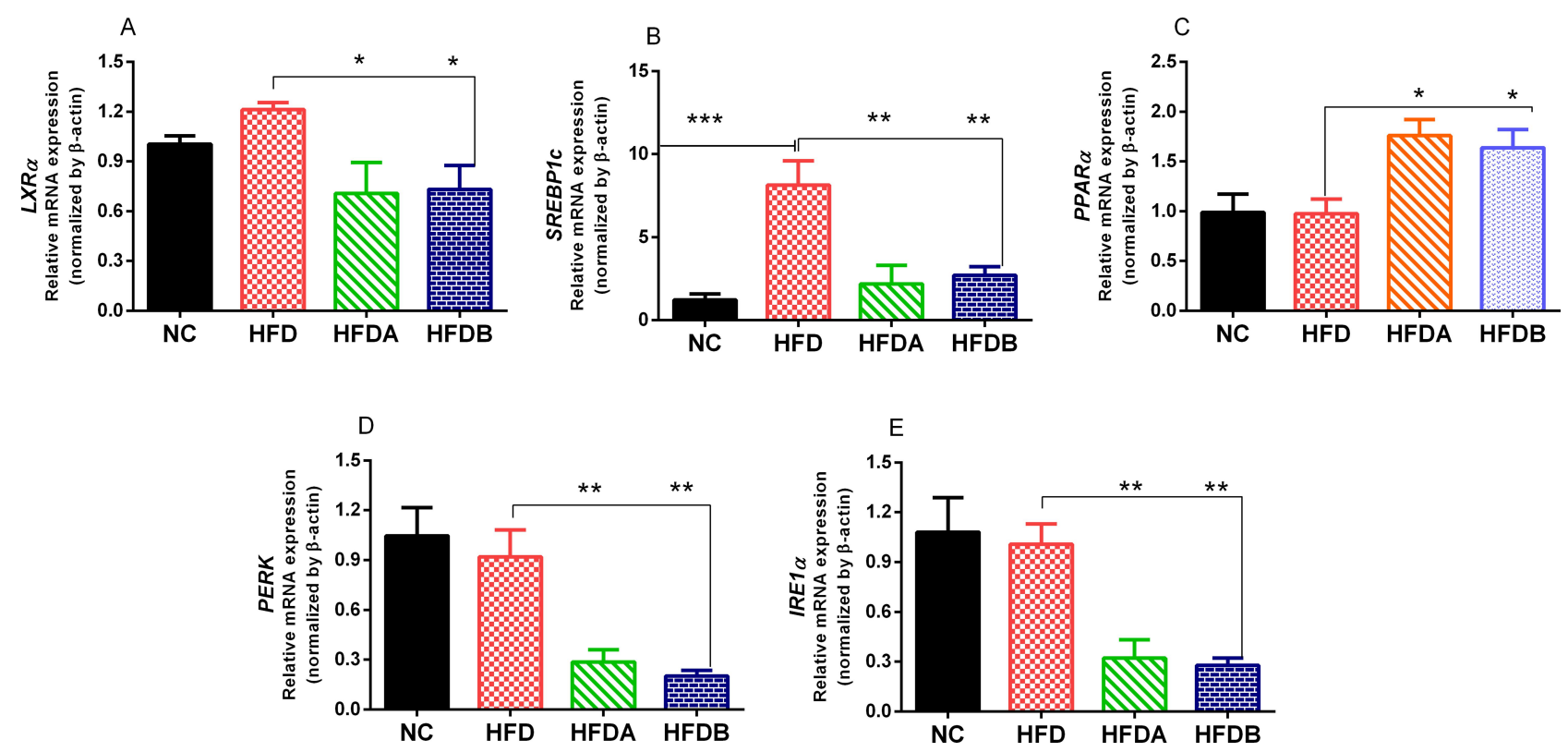

Figure 5 Effects of Uro-A and Uro-B on the expression of lipogenic and hepatic ER stress genes. Relative mRNA expression levels of LXR $\alpha$ (A), SREBPIc (B) PPAR $\alpha$ (C), PERK (D), IREI $\alpha(E)$. Data presented as mean \pm SE $(n=6)$. $*_{p}<0.05 ; *^{*} p<0.01$; *** $p<0.00$ I against HFD.
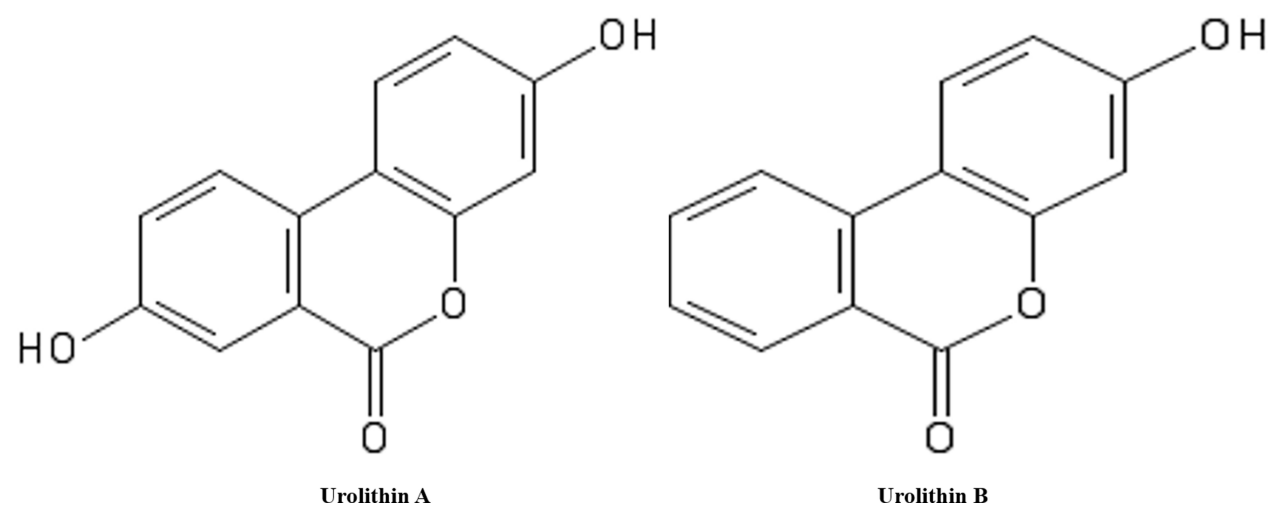

Figure 6 Chemical structure of urolithin $(\mathbf{A})$ and $(\mathbf{B})$.

transcription factors, which play an essential role in the hepatic metabolism of cholesterol and lipid. ${ }^{42}$ Similarly, sterol regulatory element-binding protein-1c (SREBP-1c) is one of a family of transcription factors, which mediates the transcription of genes involved in lipid metabolism. ${ }^{43}$ It is highly expressed in the liver and adipose tissues. However, its overexpression in the liver is associated with fatty liver disease. ${ }^{44}$ Furthermore, peroxisome proliferator-activated receptor alpha (PPAR $\alpha$ ) is a transcription factor expressed mainly in tissues associated with the catabolism of fatty acid, for example, liver, muscle, and heart. ${ }^{45}$ The increase in the catabolism of fatty acidmediated by PPAR $\alpha$ could serve as a protection for the development of obesity and its associated disorders such as non-alcoholic fatty liver disease (NAFLD). ${ }^{46}$
Our results showed that HFD feeding indeed resulted in overexpression of both LXR $\alpha$ and SREBP1c in rat liver which are partly responsible for hepatic lipid accumulation. However, treatment with both Uro-A and Uro-B significantly downregulated the expression of LXR $\alpha$ and SREBP $1 \mathrm{c}$ and thus resulted in a decrease in hepatic lipid accumulation. Conversely, urolithin treatment resulted in the upregulated expression of PPAR $\alpha$ in HFD-Uro treated rats; resulting in increased fatty acid breakdown via the beta-oxidation pathway, leading to reduced lipid accumulation. ${ }^{47}$ The counteracting effect of urolithins on the activity of LXR $\alpha$, SREBP1c, and PPAR $\alpha$ might be another mechanism through which urolithins attenuate hepatic lipid accumulation. 
The endoplasmic reticulum (ER) is an important cell organelle responsible for calcium storage, lipid synthesis, protein modification, folding, and assembly. ER stress ensued when misfolded proteins accumulate in the cell. ${ }^{48}$ To revert to the ER functional state, a series of pathways mediated by PERK (PKR-like ER kinase), IRE1 $\alpha$ (Inositol requiring enzyme $1 \alpha$ ) and ATF6 $\alpha$ (Activating transcription factor $6 \alpha$ ), known as the unfolded protein response (UPR) are activated. In the liver, unresolved ER stress is responsible for lipid accumulation causing hepatic steatosis. ${ }^{49}$ Also, the UPR pathways are upregulated in a metabolic stress state such as obesity. ${ }^{50}$ Thus, ER and indeed UPR activation function to prevent hepatic lipid accumulation and obesity development.

To determine whether these urolithins could alleviate hepatic ER stress in HFD induced-obese rats, we determined the mRNA expression levels of PERK and IRE1 $\alpha$. Indeed, Uro-A and Uro-B treatment significantly reduced mRNA expressions of PERK and IRE1 $\alpha$ when compared untreated HFD rats. Since ER stress is related to the accumulation of lipids in hepatocytes, it thus means that restoration of ER homeostasis will attenuate lipid accumulation. This result is in agreement with our histological observation (Figure 4) in which urolithin treatment reduced hepatic lipid accumulation.

\section{Conclusion}

We report here, the potential beneficial effects of Uro-A and $\mathrm{B}$ on their ability to attenuate obesity and associated symptoms. We showed that these metabolites reduced body weight gain, visceral adipose tissue mass, hepatic lipids accumulation, and oxidative stress following a highfat diet feeding through the modulation of genes associated with lipogenesis and fatty acid oxidation in addition to their ability to increase fat excretion in the feces. We equally showed that Uro-A and Uro-B have the potential to decrease hepatic ER stress through the decreased expression of PERK and IRE1 $\alpha$ which are associated with the unfolded protein response pathway. More studies are therefore required to establish urolithins as anti-obesity candidates and their potential role in mitigating hepatic steatosis and NAFLD which are both closely associated with obesity.

\section{Acknowledgment}

This project was funded by the Deanship of Scientific Research (DSR), King Abdulaziz University, Jeddah, Saudi Arabia under grant no. (KEP-15-130-41). The authors, therefore, acknowledge with thanks DSR technical and financial support. Special thanks also to the Deanship of Graduate Studies, King Abdulaziz University, Jeddah, Saudi Arabia for the award of Doctoral scholarship to Abdulrasheed O. Abdulrahman. The authors acknowledge Mohammed Kaleem, Asaad Kayali, Mahmood Dalhat, and Mohammed Razeeth Shait Mohammed for their technical assistance during the experiments.

\section{Disclosure}

The authors report no conflicts of interest in this work.

\section{References}

1. Mohamed GA, Ibrahim SR, Elkhayat ES, El Dine RS. Natural anti-obesity agents. Bulletin Faculty Pharm Cairo Univ. 2014;52 (2):269-284. doi:10.1016/j.bfopcu.2014.05.001

2. Kim JH, Kim O-K, Yoon H-G, et al. Anti-obesity effect of extract from fermented Curcuma longa $\mathrm{L}$. through regulation of adipogenesis and lipolysis pathway in high-fat diet-induced obese rats. Food Nutr Res. 2016;60(1):30428. doi:10.3402/fnr.v60.30428

3. Furukawa S, Fujita T, Shimabukuro M, et al. Increased oxidative stress in obesity and its impact on metabolic syndrome. J Clin Invest. 2017;114(12):1752-1761. doi:10.1172/JCI21625

4. Guh DP, Zhang W, Bansback N, Amarsi Z, Birmingham CL, Anis AH. The incidence of co-morbidities related to obesity and overweight: A systematic review and meta-analysis. Bmc Public Health. 2009;9(1). doi:10.1186/1471-2458-9-88

5. Word Health Organization. Obesity and Overweight; 2016. Available from https://www.who.int/news-room/fact-sheets/detail/obesity-andoverweight. Accessed October 14, 2019

6. Rodríguez-Pérez C, Segura-Carretero A, Del Mar Contreras M. Phenolic compounds as natural and multifunctional anti-obesity agents: A review. Crit Rev Food Sci Nutr. 2019;59(8):1212-1229. doi:10.1080/10408398.2017.1399859

7. Mayer MA, Hocht C, Puyó A, Taira CA. Recent advances in obesity pharmacotherapy. Curr Clin Pharmacol. 2009;4(1):53-61. doi: $10.2174 / 157488409787236128$

8. Sheba AL, Ilakkia A. Anti-obesity effect of hibiscus Sabdariffa L.- A review. Int $J$ Pharma Bio Sci. 2016;7(4). doi:10.22376/ ijpbs.2016.7.4.b341-345

9. Sun -N-N, Wu T-Y, Chau C-F. Natural dietary and herbal products in anti-obesity treatment. Molecules. 2016;21(10):1351. doi:10.3390/ molecules21101351

10. Cerdá B, Periago P, Espín JC, Tomás-Barberán FA. Identification of urolithin $\mathrm{A}$ as a metabolite produced by human colon microflora from ellagic acid and related compounds. J Agric Food Chem. 2005;53 (14):5571-5576. doi:10.1021/jf050384i

11. González-Sarrías A, Giménez-Bastida JA, García-Conesa MT, et al. Occurrence of urolithins, gut microbiota ellagic acid metabolites and proliferation markers expression response in the human prostate gland upon consumption of walnuts and pomegranate juice. Mol Nutr Food Res. 2010;54(3):311-322. doi:10.1002/mnfr.200900152

12. Savi M, Bocchi L, Mena P, et al. In vivo administration of urolithin $A$ and $B$ prevents the occurrence of cardiac dysfunction in streptozotocin-induced diabetic rats. Cardiovasc Diabetol. 2017;16 (1):80. doi:10.1186/s12933-017-0561-3

13. Espín JC, Larrosa M, García-Conesa MT, Tomás-Barberán F. Biological significance of urolithins, the gut microbial ellagic acid-derived metabolites: the evidence so far. Evid Based Complement Alternat Med. 2013;2013:1-15. doi:10.1155/2013/270418 
14. García-Muñoz C, Hernández L, Pérez A, Vaillant F. Diversity of urinary excretion patterns of main ellagitannins' colonic metabolites after ingestion of tropical highland blackberry (Rubus adenotrichus) juice. Food Res Int. 2014;55:161-169. doi:10.1016/j.foodres.2013.10.049

15. Cerdá B, Espín JC, Parra S, Martínez P, Tomás-Barberán FA. The potent in vitro antioxidant ellagitannins from pomegranate juice are metabolised into bioavailable but poor antioxidant hydroxy-6H-dibenzopyran-6-one derivatives by the colonic microflora of healthy humans. Eur J Nutr. 2004;43(4):205-220. doi:10.1007/s00394-004-0461-7

16. Seeram NP, Aronson WJ, Zhang Y, et al. Pomegranate ellagitannin-derived metabolites inhibit prostate cancer growth and localize to the mouse prostate gland. J of Agri Food Chem. 2007;55 (19):7732-7737. doi:10.1021/jf071303g

17. Al-Muammar MN, Khan F. Obesity: the preventive role of the pomegranate (Punica granatum). Nutrition. 2012;28(6):595-604. doi:10.1016/j.nut.2011.11.013

18. Woods SC, Seeley RJ, Rushing PA, D’Alessio D, Tso P. A controlled high-fat diet induces an obese syndrome in rats. $J$ Nutr. 2003;133 (4):1081-1087. doi:10.1093/jn/133.4.1081

19. Yang C, Li LH, Yang LG, Lu H, Wang SK, Sun GJ. Anti-obesity and Hypolipidemic effects of garlic oil and onion oil in rats fed a high-fat diet. Nutr Metab. 2018;15.

20. Vanzo A, Vrhovsek U, Tramer F, Mattivi F, Passamonti S. Exceptionally fast uptake and metabolism of cyanidin 3-glucoside by rat kidneys and liver. J Nat Prod. 2011;74(5):1049-1054. doi:10.1021/np100948a

21. Bradford MM. A rapid and sensitive method for the quantitation of microgram quantities of protein utilizing the principle of protein-dye binding. Anal Biochem. 1976;72(1-2):248-254. doi:10.1016/0003-2697(76)90527-3

22. Aebi H. Catalase in Vitro. In: Methods in Enzymology. Vol. 105. Elsevier; 1984:121-126.

23. Li X. Improved pyrogallol autoxidation method: a reliable and cheap superoxide-scavenging assay suitable for all antioxidants. J Agric Food Chem. 2012;60(25):6418-6424. doi:10.1021/jf204970r

24. Ohkawa H, Ohishi N, Yagi K. Assay for lipid peroxides in animal tissues by thiobarbituric acid reaction. Anal Biochem. 1979;95 (2):351-358. doi:10.1016/0003-2697(79)90738-3

25. Folch J, Lees M, Stanley G. A simple method for total lipid extraction and purification. J Biol Chem. 1957;226(1):497-509.

26. Livak KJ, Schmittgen TD. Analysis of relative gene expression data using real-time quantitative PCR and the $2-\Delta \Delta \mathrm{CT}$ method. methods. 2001;25(4):402-408. doi:10.1006/meth.2001.1262

27. Shin J-W, Seol I-C, Son C-G. Interpretation of animal dose and human equivalent dose for drug development. J Korean Oriental Med. 2010;31(3):1-7.

28. Hwang NH, Woo SL. Frontiers in Biomedical Engineering: Proceedings of the World Congress for Chinese Biomedical Engineers. Springer Science \& Business Media; 2003.

29. Tomás-Barberán FA, González-Sarrías A, García-Villalba R, et al. Urolithins, the rescue of "old" metabolites to understand a "new" concept: metabotypes as a nexus among phenolic metabolism, microbiota dysbiosis, and host health status. Mol Nutr Food Res. 2017;61 (1):1500901. doi:10.1002/mnfr.201500901

30. Echeverria F, Valenzuela R, Bustamante A, et al. Attenuation of High-Fat Diet-Induced Rat Liver Oxidative Stress and Steatosis by Combined Hydroxytyrosol- (HT-) Eicosapentaenoic Acid Supplementation Mainly Relies on HT. Oxid Med Cell Longev. 2018;2018:5109503. doi:10.1155/2018/5109503

31. Abarca-Gómez L, Abdeen ZA, Hamid ZA, et al. Worldwide trends in body-mass index, underweight, overweight, and obesity from 1975 to 2016: a pooled analysis of 2416 population-based measurement studies in 128. 9 million children, adolescents, and adults. Lancet. 2017;390(10113):2627-2642.

32. Rodriguez-Ramiro I, Vauzour D, Minihane A. Polyphenols and non-alcoholic fatty liver disease: impact and mechanisms. Proce Nutrition Soc. 2016;75(1):47-60. doi:10.1017/S0029665115004218
33. Pandey KB, Rizvi SI. Plant polyphenols as dietary antioxidants in human health and disease. Oxid Med Cell Longev. 2009;2 (5):270-278. doi:10.4161/oxim.2.5.9498

34. Sayed S, Ahmed M, El-Shehawi A, et al. Ginger Water Reduces Body Weight Gain and Improves Energy Expenditure in Rats. Foods. 2020;9(1):1. doi:10.3390/foods9010038

35. Cohen JC, Horton JD, Hobbs HH. Human fatty liver disease: old questions and new insights. Science. 2011;332(6037):1519-1523. doi:10.1126/science. 1204265

36. Blokhina O, Virolainen E, Fagerstedt KV. Antioxidants, oxidative damage and oxygen deprivation stress: a review. Ann Bot. 2003;91 (2):179-194. doi:10.1093/aob/mcf118

37. Svegliati-Baroni G, Candelaresi C, Saccomanno S, et al. A model of insulin resistance and nonalcoholic steatohepatitis in rats: role of peroxisome proliferator-activated receptor- $\alpha$ and n-3 polyunsaturated fatty acid treatment on liver injury. Am J Pathol. 2006;169 (3):846-860. doi:10.2353/ajpath.2006.050953

38. Kang I, Kim Y, Tomás-Barberán FA, Espín JC, Chung S. Urolithin A, $\mathrm{C}$, and $\mathrm{D}$, but not iso-urolithin $\mathrm{A}$ and urolithin $\mathrm{B}$, attenuate triglyceride accumulation in human cultures of adipocytes and hepatocytes. Mol Nutr Food Res. 2016;60(5):1129-1138. doi:10.1002/ mnfr.201500796

39. Zhao W, Wang L, Haller V, Ritsch A, Novel A. Candidate for Prevention and Treatment of Atherosclerosis: urolithin B Decreases Lipid Plaque Deposition in apoE-/- Mice and Increases Early Stages of Reverse Cholesterol Transport in ox-LDL Treated Macrophages Cells. Mol Nutr Food Res. 2019;63(10):1800887. doi:10.1002/ mnfr.201800887

40. Lei F, Zhang X, Wang W, et al. Evidence of anti-obesity effects of the pomegranate leaf extract in high-fat diet induced obese mice. Int J Obes. 2007;31(6):1023. doi:10.1038/sj.ijo.0803502

41. Drent ML, van der Veen EA. First clinical studies with orlistat: a short review. Obes Res. 1995;3(Suppl 4):623S-625S. doi:10.1002/ j.1550-8528.1995.tb00236.x

42. Dib L, Bugge A, Collins S. LXR $\alpha$ fuels fatty acid-stimulated oxygen consumption in white adipocytes. J Lipid Res. 2014;55(2):247-257. doi:10.1194/jlr.M043422

43. Strable MS, Ntambi JM. Genetic control of de novo lipogenesis: role in diet-induced obesity. Crit Rev Biochem Mol Biol. 2010;45 (3):199-214. doi:10.3109/10409231003667500

44. Knebel B, Haas J, Hartwig S, et al. Liver-specific expression of transcriptionally active SREBP-1c is associated with fatty liver and increased visceral fat mass. PLoS One. 2012;7(2):e31812. doi:10.1371/journal.pone.0031812

45. Ferré P. The biology of peroxisome proliferator-activated receptors: relationship with lipid metabolism and insulin sensitivity. Diabetes. 2004;53(suppl 1):S43-S50. doi:10.2337/diabetes.53.2007.S43

46. Yeh Y-T, Cho -Y-Y, Hsieh S-C, Chiang A-N. Chinese olive extract ameliorates hepatic lipid accumulation in vitro and in vivo by regulating lipid metabolism. Sci Rep. 2018;8(1):1057. doi:10.1038/ s41598-018-19553-1

47. Hashimoto T, Cook WS, Qi C, Yeldandi AV, Reddy JK, Rao MS. Defect in peroxisome proliferator-activated receptor $\alpha$-inducible fatty acid oxidation determines the severity of hepatic steatosis in response to fasting. J Biol Chem. 2000;275(37):28918-28928. doi:10.1074/jbc. M910350199

48. Hampton RY. ER stress response: getting the UPR hand on misfolded proteins. Current Biol. 2000;10(14):R518-R521. doi:10.1016/S09609822(00)00583-2

49. Rutkowski DT, Wu J, Back S-H, et al. UPR pathways combine to prevent hepatic steatosis caused by ER stress-mediated suppression of transcriptional master regulators. Dev Cell. 2008;15(6):829-840. doi:10.1016/j.devcel.2008.10.015

50. Özcan U, Cao Q, Yilmaz E, et al. Endoplasmic reticulum stress links obesity, insulin action, and type 2 diabetes. Science. 2004;306 (5695):457-461. doi:10.1126/science. 1103160 


\section{Publish your work in this journal}

Diabetes, Metabolic Syndrome and Obesity: Targets and Therapy is an international, peer-reviewed open-access journal committed to the rapid publication of the latest laboratory and clinical findings in the fields of diabetes, metabolic syndrome and obesity research. Original research, review, case reports, hypothesis formation, expert opinion and commentaries are all considered for publication. The manuscript management system is completely online and includes a very quick and fair peer-review system, which is all easy to use. Visit http://www.dovepress.com/testimonials.php to read real quotes from published authors. 